\title{
Framework for Interactive Million-Neuron Simulation
}

\author{
Running title: Interactive Million Neuron Simulation \\ Mark Hereld, ${ }^{1,2}$ Rick L. Stevens, ${ }^{1,2}$ Wim van Drongelen, ${ }^{2,3}$ \\ and Hyong C. Lee ${ }^{3}$ \\ ${ }^{1}$ Mathematics and Computer Science Division, Argonne National Laboratory, Argonne, IL \\ ${ }^{2}$ Computation Institute, The University of Chicago, Chicago, IL \\ ${ }^{3}$ Department of Pediatrics, The University of Chicago, Chicago, IL
}

\section{Contact Information:}

Mark Hereld

Argonne National Laboratory,

9700 S. Cass Ave., Argonne, IL 60637-1470.

Tel: (630) 2524170

Fax: (630) 2524786

E-mail: hereld@mcs.anl.gov

Presented during the international conference "An Overview of Epilepsy Research: What, Where, When and Why," organized by the Center for Integrative Neuroscience and Neuroengineering Research (CINNR), May 19-20, 2006, in Chicago. 


\begin{abstract}
Large simulations have become increasingly complex in many fields, tending to incorporate scaledependent modeling and algorithms and wide-ranging physical influences. This scale of simulation sophistication has not yet been matched in neuroscience. In this paper we describe a framework aimed at enabling natural interaction with complex simulations: their configuration, initial conditions, monitoring, and analysis. The architecture is built on three cornerstone components: active probes, adaptive data capture, and visual interface. The resulting synthesis will enable interactive exploration of live simulations running on supercomputing platforms.
\end{abstract}

\title{
Keywords:
}

Computer simulation, Realistic modeling, Parallel computation, Neocortex, Epilepsy. 


\section{A Introduction}

Brain electrical activity is generated by an immense network of billions of neurons. Because of the brain's complexity, brain electrical activity has been studied either at the microscopic level, ranging from ion channels and single cells to small networks of cells, or at macroscopic level, in clinical measurement such as electroencephalogram. Between these two extremes lie complex interactions between interconnected populations of cells, interactions that are opaque to current experimental techniques. Detailed understanding of brain function and dysfunction will require multiscale simulations to span these modeled domains: (1) employing different data models, communication patterns, and algorithms as appropriate to each layer of the simulation, (2) adaptively deploying corresponding simulation threads, and (3) matching results obtained at these different scales.

Also, as the components of these simulations are developed within the computational neuroscience community, the need for a framework for testing performance scaling and verification of integrated multiscale models will become critical for several reasons: (1) understanding simulation performance scaling is prerequisite to knowing what scientific questions are practical to pursue, (2) understanding performance is critical to simulation planning activities and resource allocation, and, (3) increasingly complex simulations demand sophisticated parameter configuration management.

We describe a framework for interactive simulation and exploration of multiscale models. The framework includes components to enable interactive simulation management, real-time simulation state visualization and steering, and final result visualization. Because there are no standard methods for probing, visualizing, and manipulating large simulations across scales, this model will be of interest to the broader simulation community.

We organize the paper into two main sections. In the first section we describe the proposed architecture and the three key components of its framework: leaky bucket stream archiving, active probes, and image-based modeling and configuration. In the second section we describe several processes 
enabled by the framework: model overlay visualization, interactive playback, and live performance modeling.

\section{B Related Work}

Large simulations have become increasingly complex in many fields, tending to incorporate scaledependent modeling and algorithms and wide-ranging physical influences (Calder et al. 2002). This scale of simulation sophistication has not yet been matched in neuroscience.

In simulations of biological neural networks, parallel computing has expanded the scale and complexity of feasible systems (e.g., Migliore et al. 2006). Support for modeling realistic neurons and networks on such platforms has been codified in domain-specific scripting environments such as pGENESIS (Goddard and Hood 1997; Bower and Beeman 1998; pGENESIS 2005). Still, the largest simulations in terms of cell count have been achieved with simplified models of the integrate-and-fire sort

- the latest of which appears to be a test at the 100 billion cell mark carried out on a 27 -node Beowulf cluster over 50 days (Izhikevich 2005). The Blue Brain Project targets simulation of a neocortical column comprising some 10,000 cells on IBM's Blue Gene/L computer platform (Blue Brain 2005).

Interactive high-performance computing has been actively pursued in several contexts. Successful experiments in visualization of simulations with interactive user input and computational steering have a long history (Canfield et al. 1996; Diachin et al. 1996; Matlab (Mathworks 2005) has attracted research into parallel scientific computing (Kepner 2004; Choy and Edleman 2005) because it is flexible, fluid, and familiar. Nonetheless, high-performance computing continues to lean heavily-indeed, almost exclusively—on batch scheduling for job execution.

Simulations of large complex neurons have led to visualizations of the 3D physical models (Leigh et al. 1993), demonstrating the translation of simulation configuration to model visualization. Highperformance visualization tools for more general parallel visualization applications have become available (ParaView 2005) but have not been applied to biological neural network simulations. 
High-quality performance characterization tools are available for parallel platforms (Mohr et al. 1994; Tau 2005), but none enable interactive exploration of potentially live simulations.

Our own research in these areas includes the following.

Neocortex model and simulation - We have conducted preliminary work (Hereld et al. 2004) to develop a first-generation detailed and scalable model of neocortex (Van Drongelen et al. 2002, 2004a, 2004b, 2005). This model is based on details of neocortical histology; that is, the neural elements are arranged according to horizontal layers and vertical columns (see, e.g., Nieuwenhuys 1994; Mountcastle, 1997; DeFelipe et al. 2002). We represented neocortical cell types by multicompartmental models, in which different parts of the cell are modeled by cylindrical compartments; the size and arrangement of the compartments are constrained by the morphology of real neurons. Six major cell types are included in the current model: superficial pyramidal, deep pyramidal, chandelier, and three types of basket cells. The basket cells are further divided into several subtypes, each governed by somewhat different internal state parameters. The basic equation governing the behavior of the compartments is a discrete implementation of the cable equation with modified Hodgkin and Huxley-type ion channels. The neurons are placed in a 3D grid and connected to each other by using connectivity rules derived from connections in the mammalian cortex (e.g., Nieuwenhuys 1994; Feldmeyer and Sakmann 2000; Hellwig 2000; Krimer and Goldman-Rakic 2001). Simulation time is stepped uniformly and synchronously. Spikes are represented as events that communicate an instantaneous change at the input of a synapse, with weight and delays programmed into each connection.

The computational model has been implemented in parallel GENESIS (Bower and Beeman 1998) to facilitate a high degree of detail, large-scale simulations and multiple parameter searches. A scalable version of the model runs on the Jazz computing cluster at Argonne National Laboratory, where we performed $>10$ years of single-processor simulation within a two-year period. Details of how the model is constructed and preliminary results obtained with our approach have been published (Van Drongelen et al. 2002, 2004a, 2004b, 2005). More recently we have run our simulation on the Blue Gene/L machine at 
Argonne National Laboratory, testing the model's performance characteristics on 32 to 512 nodes (Hereld et al. 2005).

The resulting model of the neocortex is being studied as a platform for understanding the onset of epileptiform activity in children. The goal of the research program is to understand epileptic activity in the neocortex well enough to provide clues about its cause and possible cures. At present the model can generate spontaneous and evoked activity at the compartmental, cellular, and network scales. The compartmental and cellular activity consists of complete knowledge of the local membrane potential and current. The extracellular activity was obtained as a weighted sum of currents generated by the model neurons (Nunez 1981). Exciting recent results demonstrated that weakly coupled networks can sustain bursting activity in neural populations (Van Drongelen et al. 2005); a surprising counterintuitive result has also been found in real cortical networks (Pumain et al. 1985; Feng and Durand 2004, 2005; van Drongelen et al., 2005). While our preliminary studies demonstrate the feasibility of modeling extracellular local field potentials, no model currrently can connect local field potentials across multiple scales to cellular and molecular parameters systematically. Further development is needed of a multiscale neural model associated with adequate parameter evaluation tools.

Scalable simulation code - Although we continue to use this code to develop our neocortex model, the implementation does not scale beyond about 100,000 cells. For this reason, and anticipating the fundamentally different needs of future simulations, we began to develop a simulation code, called pNeo, that can be optimized for much larger runs (Hereld et al., 2004). The new code has been tested on up to 250 nodes of the Argonne Jazz cluster in simulations of over 100,000 cells (Hereld et al., 2005). It has also been run on 512 nodes of the Argonne Blue Gene/L machine in simulations of over twice that size. Recent results from these experiments show near-linear speedup to $\mathrm{P}=256$ processors for fixed problem small enough to run on a few processors (Fig. 1). Memory limitations constrain problem size at low P. For the current code, larger problems will continue to exhibit good speedup on the available 512 nodes. Interactive visualization - For visualizing, interacting with, and navigating through the large, complex, multidimensional datasets to be created by this project, we have developed high-resolution, high- 
performance display systems, built using arrays of commodity projectors and driven by clusters of commodity computers (Hereld et al. 2000). These systems present tens of millions of pixels to groups of interacting research collaborators. In addition to the hardware technologies, this effort has resulted in a substantial application development toolkit.

To test the experience of interacting with dense visualizations of complex systems, we have begun experiments on the ActiveMural, our room-sized high-resolution display system (Fig. 2). These experiments are based on a dense diagram of a metabolic pathway model. The application spatially codes the image area with clickable resources: supplementary data on proteins and reactions in local databases, browser launching with encapsulated queries to large public databases, and 3D viewing application launching for structure analysis.

\section{Architecture and Three Core Components}

Increasingly sophisticated simulations will require complex configuration and initialization. And with this complexity comes uncertainty. Already, large simulations are generating huge datasets requiring analysis and interpretation. Often, most of the data generated during a simulation must be discarded because of lack of storage space, bandwidth, or both. Simulations on expensive computational resources must therefore be configured in advance, with potentially risky choices of what to save and what to discard. Additionally, planning simulation sequences requires a priori knowledge of the performance properties in order to avoid wasting precious resources on runs that are destined to fail because of limitations of memory, disk space, or job time.

Our own neocortex simulations have pressed on all of these issues. We are developing a framework that is designed to obviate these problems and that represents what we believe to be a vision of the future of large scale complex multiscale simulations. Figure 3 shows a schematic of the key features of our framework. In it the simulation is represented in the upper left corner, running on a supercomputing platform. The simulation is configured in the usual way, with little or no modification to existing coding strategies - represented here by a block of configuration information that can be rolled in at the start of 
the simulation. The collection of data and performance information from the simulation is managed by a system that we call active probes. These probes provide the means for expressing what data is to be captured for storage or monitoring. The data and performance capture policy controls which streams are allowed to escape the execution cocoon of the simulation. Some elements of this capture policy can be adjusted in time (or real time) through changes in the priority, patterned sampling, trigger, and interactive selection mechanisms. The user views the simulation data streams using a playback metaphor, which considers probe streams in the same way that a multitrack audio system deals with audio streams.

Visualization of the progress of the simulation is presented in images generated from the model and configuration information. These same visual representations provide the canvas on which simulation configuration information and active probe selection and activation are managed.

The interfaces to the simulation and to the user are separated by a line that captures the different possible relationships between them: real time, buffer delayed, or offline. If the simulation is running on a platform that supports interactive job execution, then the user will be able to work directly with the simulation through the interface. In some cases, job execution might not be quite interactivearchitectures that introduce significant latency and Grid applications that entrain a changing collection of computational resources are possible candidates; in such cases, the data stored in the buffer can be worked with as it becomes available. In other cases, typical in supercomputing applications, batch execution may force the data to be stored and viewed after the job has completed and the data has been staged off the machine. Our design enables real-time interactive computing but does not require it.

\section{C.1 Late Commitment and the Leaky Bucket}

Large simulations notoriously generate more data than can be efficiently stored, and so researchers are typically forced to select in advance the data that they anticipate will have the most scientific value. Testing the behavior of a simulation to understand what computed quantities are most interesting often requires multiple complete simulations. These issues have led us to consider a model that allows the user

to defer commitment to a particular subset of data until the last moment and that favors rapid exploration 
of the simulation behavior in aid of intuition building. This "leaky bucket" model for adaptively regulating data archiving is illustrated in Fig. 4.

At any time, a set of explicit selection criteria are in effect, and the user chooses these interactively as the simulation proceeds. Data created by the simulation is tagged and timestamped so that later reevaluation in light of changed selection criteria can be used to discard less interesting data as storage limits are reached. By this means an adaptive system of prioritization, responsive to the user's evolving understanding of the simulation results, can drive the continuous balancing of data needs against available storage space.

\section{C.2 Active Probes}

Our idea of active probes captures the notion that monitoring signals from a simulation should be as simple as attaching a sensor to a physical object. These probes then generate streams of data representing the quantities under observation. We want this interface to the simulation to include several kinds of measurement, of which the following are typical examples:

- Single state variable: the action potential of a particular cell

- Class of object variables: the action potential of each basket cell

- Filtered: the mean value of action potential of superficial pyramidal cells over a patch area

- Triggered: the action potential of a particular cell whenever another cell fires

- Performance measure: allocated memory

Because we think of these implementations in a physical way analogous to an oscilloscope probe, and because the framework lets us interact with a visualization of the model, we enable natural modes of interacting with the simulation.

The central question in the implementation of active probes is, How can we provide access to the variables instantiated in all sorts of ways (static, scoped, register, intermediate) to the capture selector in a way that requires communication only of selected quantities? A second question is, Where do we best implement filters, combinations, and trigger tests without introducing unnecessary computation or 
communication? Note that because we want to enable interactive selection of active probes their activation must be made at run time.

Two approaches to implementing active probes look promising. The first leverages the symbol table created by the compiler to gain access to variable names and memory locations. Additionally, a few function calls would be introduced into the code to synchronize the efforts of the capture selector with the simulation loop.

The second approach is built around manual instrumentation of the simulation code, with macros to identify values that might be probed and a few functions that implement the interface between the simulation and the capture selector. Automated instrumentation techniques have been developed elsewhere (e.g., Mohr et al. 1994) and could be leveraged later. These macros would express the circumstances of each type of variable that must be handled (intermediate variables, indexed quantities, filtered quantities, triggers) and manage registration of symbols and generation of the activation flag list at compile time. A schematic of the process is outlined in Table 1.

In both cases additional function calls would be required in the simulation to support the processing of active probe data, mainly through the inclusion of processcaptureselector () in the simulation loop and the inclusion of initActiveprobes () at the outset of the simulation. Filters and triggers would be implemented in the processing of the posted quantities depending on the conditions set by the data and performance capture policy. In this way the application programmer would not be burdened with definition, instantiation, and instrumentation of a wide range of possible filtering techniques. Instead, these would be instantiated and managed in the shared code contained in the capture selector and controlled by the capture policy unit (see Fig. 3).

Whereas the symbol table approach involves less modification to the code, it can meaningfully expose only quantities that are held in variables that persist to the end of the time step. The macro instrumentation approach enables a wider range of values for export, at the expense of more invasive embellishments to the code. The final implementation will likely combine features from both approaches. 


\section{C.3 Image-based Modeling and Simulation Setup}

Images, as opposed to algorithms, can often represent complex features in a straightforward and intuitive way. Visual representation of the simulation model can provide the substrate on which at least two distinct and complementary activities can take place:

- the user interactively selects and assigns configuration state information, and

- the user reviews displayed configuration state - population densities, scalar parameter fields, spike activity fields.

Each rendered representation would be automatically generated from the model and current configuration. Some of these visualizations would be straightforward to compute, as would be the case for the overall map of cell placement in two or three dimensions. Hierarchically organized groupings of subnetworks would also yield to simple techniques, so long as the interconnections were not labyrinthine. Wiring diagram layout, however, is in general a difficult problem. We can rely initially on well-studied methods such as force-directed layout algorithms (Tutte 1963; Fruchterman and Reingold 1991).

With cell types, connection types, and spatial layout available in graphical form, one can create richly differentiated and expressive selection sets. We envision a wide variety of powerful configuration methods to become possible with this toolset. The following are examples of these interactively driven parametric configuration techniques:

- Graphical programming of probability density function can be expressed as a function of radius or a 2D density map painted by the user or can be extracted from an image.

- Similar techniques can be applied for specifying variation of other quantities across spatial or temporal dimensions.

- Parameter groups can be set over a region of interest selected by using the cursor lasso.

- Parameter groups can be copied from one region and pasted to another.

- Smooth variations can be computed and assigned for transition regions. 
In order to implement these methods, a large number of techniques developed for word processing, page layout, photographic image manipulation, and illustration can be borrowed.

Because complex configuration state can be expressed fluidly and quickly with this interface, and because it will be possible in some cases to interact with a running simulation, a new capability becomes possible: on-the-fly reconfiguration of the simulation. This capability will significantly enhance our ability to quickly sort through the relationship between the model (and its configuration) and the behavior of the simulation. Not a substitute for detailed parameter searches, this approach instead enables a rapid skimming of possibilities and intuition building - an excellent tool for exploration. Likewise, this capability can be employed during performance characterization and application performance modeling by enabling rapid testing of dependencies and quick measurements of effects.

\section{Consequences of This Approach to Interactive Simulation}

This framework, which couples visualization of simulation streams and performance streams from a system of active probes to representations of the system model, creates an environment for fluid, flexible, and natural interaction with complex and sophisticated simulations. It provides a natural mechanism for integrated performance modeling, configuration, and reconfiguration of the simulation and the potential for monitoring and steering the simulation.

A number of capabilities will be enabled, capabilities not currently available but likely to become increasingly attractive with complex multiscale simulations: rapid configuration and testing to support strategic planning and management of flocks of simulation sets; real time, buffer-delayed, or offline interactive modalities; and sophisticated data-capture management based on priority, patterns, triggers, real time steering, and resource limits.

\section{D.1 Model Overlay Visualization}

To facilitate interactive manipulation and interpretation of the state of the simulation, we envision an environment based on visual representation of the simulation model, decorated with live data showing 
animations of the active probe values. Simulations are often best understood with visual representations of the model at different scales. Consequently we are motivated to create symbolic visualizations of many aspects of the simulation: internal cell model parameterization, wiring schematic with interconnection probability distributions, overall cell layout, and regional maps to capture interacting subnetworks.

These representations will be derived automatically from the simulation configuration files, which in our current code already express most of the necessary characteristics. This direct relationship between configuration files and visual representation is critical to enabling interactive control and monitoring of the simulation. It is also critical to streamlining the development process by leveraging the existing syntax of our simulation configuration (based originally on the GENESIS neural simulation environment). Specifically, we plan to organize configuration and the generated visual representations around three descriptive layers:

- Individual cell models will be organized by cell type. Parameters governing the evolution of the internal state will be configured and monitored. These can be varied by cell, by cell type, or by parametric rules (e.g., spatial gradients, random variation).

- Subnetworks will be laid out in space and wired according to parametric rules.

- Groups of subnetworks will be laid out in space and interconnected according to parametric rules.

An example is shown in Fig. 5. The scenario depicted in this mockup illustrates several aspects of the proposed interface: a deep pyramidal cell class has been selected (solid line circle), and instances are shown as filled dots - a popup presents the opportunity to modify cell class parameters; a region of interest has been selected (black dashed annulus). Note that the ROI intersects several processors; active probes (numbered pointers) have been placed to capture mean action potential in the annulus and action potential of a single cell. This example illustrates a few of the basic notions to be explored in the project as they might apply to simple selections of small numbers of cells or connections. 


\section{D.2 Multitrack Playback Metaphor}

As with interactive visual configuration, the visual representation of the simulation model provides a canvas on which the user specifies, selects, manipulates, activates, and deactivates active probe sets. The deployment of the probes affect the flow of data from a live simulation as well as the data presented to the user at run time or during offline review and analysis. Several key aspects of the architecture enable new and useful ways to manage the capture of useful data from large complex simulations.

First, data from the simulation is stored to disk with the understanding that some or all of it may be overwritten as the user refines the priorities interactively. The only a priori constraints are bandwidth to disk and total available disk, defining how much data can be exported from the simulation. In contrast, with the conventional approach, the bandwidth and space limits force choices that are made at the outset of the simulation and remain fixed until the simulation ends.

Second, active probes enable rapid and interactive modification of the data selected for review and storage. This feature enables live tuning of the priorities as the behavior of the simulation is actively studied.

Third, with the ability to rapidly reconfigure the simulation, it is easy to configure the simulation to multiplex data from several probe sets onto the limited bandwidth channel. Several variables can be sampled at lower time resolution, for example alternating at each simulation time step.

Fourth, trigger conditions can be added to the probe set selection criteria. In this way, once identified and codified by a trigger condition, interesting features of the simulation can be captured without storing less interesting prelude and postlude data. And because the architecture buffers the data, the triggers can be quite sophisticated and involve look-ahead evaluation.

The proposed architecture therefore encourages an interesting new strategy. The user begins by streaming as much data as possible to disk, subject only to the same constraints suffered by the conventional approach. As the simulation proceeds, the user can adjust priorities and probe set depending on the results from the simulation. Over time, the optimal data collection scheme (probes sets, switching 
patterns, priorities) is impressed on the interface to the simulation. The earlier "misguided" or naïve priorities haven't cost anything — less important data will be overwritten by more important data.

\section{D.3 Live Performance Modeling}

The proposed framework also enables a mode of performance characterization that complements available tools. In particular, it will encourage rapid and intuitive hypothesis-driven exploration of the performance properties of the simulation by enabling interactive and selective study of performance metrics to capture critical behavior and resource usage patterns. This feature contrasts with the traditional approach of instrumenting code to log every quantity that one might find useful, running the simulation, sifting through the resulting logs (sometimes with the aid of visualizations), reinstrumenting the code to catch additional performance data, and iterating until the performance model emerges.

Generally, the primary goal of these explorations is to extract a model for the performance scaling relating execution cycles, processor count, memory requirements, network bandwidth, and I/O bandwidth to problem size and configuration. The features of the present framework will largely suffice to support live performance modeling.

\section{E Concluding Remarks}

Despite the current knowledge of cortical structure and functions, the relationship between neural activities across scales in the nervous system is poorly understood. This lack of understanding is largely due to current experimental techniques in neuroscience, which are limited to detailed, small-scale electrophysiological recordings from small groups of neuron or to averaging measurements of very large networks, lacking the required detail. The implication of this limitation is that no current experimental technique can adequately correlate the behavior at the two extremes.

We have described a framework for interactive simulation that combines an adaptive scheme for archiving simulation data streams, a flexible and natural data selection architecture that is based on the metaphor of the probe, and incorporation of visual techniques into both configuration and monitoring 
processes. We have argued that applying such a framework to complex simulations running on supercomputers will enable a fundamentally different and better platform for exploration.

Taming complex configuration, management, and understanding of multiscale simulations by packaging the complexity in interactive visual form creates a more accessible interface to the inner workings of the system under study and to the scientific results, while it enhances the exploratory nature of the scientific process. Clearly the framework described in this paper could extend beyond research in neuroscience, to any field that engages high-performance computing to explore complex systems through simulation: nanoscience, civil engineering, climatology, hydrology, systems biology, and more.

\section{Acknowledgments}

This work was supported by the U.S. Department of Energy under Contract W-31-109-Eng-38. We thank Drs. M. H. Kohrman, J.-M. Ramirez, and V. L. Towle for discussion and useful comments. We thank the Falk Foundation and the Linn Family for support.

\section{F References}

Blue Brain Project. 2005. http://bluebrainproject.epfl.ch/Blue\%20Brain\%20Project.htm.

Bower JM, Beeman D. 1998. The book of GENESIS. New York: Springer-Verlag.

Butera RJ, Rinzel J, Smith JC. 1999. Models of respiratory rhythm generation in the pre-

Botzinger complex, I: bursting pacemaker neurons. J Neurophysiol 82:382-397.

Calder AC, Fryxell B, Plewa T, Rosner R, Dursi LJ, Weirs VG, Dupont T, Robey HF, Kane JO, Remington BA, Drake RP, Dimonte G, Zingale M, Timmes FX, Olson K, Ricker P, MacNeice P, Tufo HM. 2002. On validating an astrophysical simulation code. ApJS 143 
Canfield T, Diachin D, Freitag L, Heath D, Herzog J, Michels W. 1996. Interactive computational models of particle dynamics using virtual reality. In: Proceedings of the symposium on virtual reality in manufacturing research and education; Chicago. p. 80-88.

Choy R, Edelman A. 2005. Parallel MATLAB: doing it right. In: Proceedings of the IEEE; February.

DeFelipe J, Alonso-Nanclares L, Arellano JI. 2002. Microstructure of the neocortex: comparative aspects. J Neurocytol 31:299-316.

Diachin D, Freitag L, Heath D, Herzog J, Michels W, Plassmann P. 1996. Interactive simulation and analysis of emission reduction systems for commercial boilers. In: Proceedings of the SCS '96 multiconference; April; p. 352-357.

Elsen FP, Ramirez JM. 1998. Calcium currents of rhythmic neurons recorded in the isolated respiratory network of neonatal mice. J Neurosci 18:10652-1062.

Feldmeyer D, Sakmann B. 2000. Synaptic efficacy and reliability of excitatory connections between principal neurons of the input (layer 4) and output (layer 5) of the neocortex. J Physiol 525:31-39.

Feng Z, Durand DM. 2004. Suppression of excitatory synaptic transmission can facilitate lowcalcium epileptiform activity in the hippocampus in vivo. Brain Res 1030:57-65.

Feng Z, Durand DM. 2005. Propagation of low-calcium non-synaptic induced epileptiform activity to the contralateral hippocampus in vivo. Brain Res 1005:25-35. 
Fruchterman T, Reingold E. 1991. Graph drawing by force-directed placement. Softw - Pract Exp 21(11):1129-1164.

Goddard NH, Hood G. 1997. Parallel GENESIS for large scale modeling. In: Computational neuroscience: trends in research 1997. New York: Plenum. p. 911-917.

Hellwig B. 2000. A quantitative analysis of the local connectivity between pyramidal neurons in layer 2/3 of the rat visual cortex. Biol Cybern 82:111-121.

Hereld M, Judson, IR, Stevens RL. 2000. Tutorial: introduction to building projection-based tiled display systems. IEEE Computer Graphics and Applications 20(4):22-28.

Hereld M, Stevens RL, Van Drongelen W, Lee HC. 2004. Developing a petascale neural simulation. Invited presentation Joint Meeting of 5th International Conference on Bioelectromagnetism and 5th International Symposium on Noninvasive Functional Source Imaging within the Human Brain and Heart (BEM\&NFSI), Minneapolis, Minnesota, May 2005. IEEE Catalog No: 04CH37558C, ISBN: 0-7803-8440-7: 3999-4002.

Hereld M, Stevens R, Teller J, van Drongelen W, Lee H. 2005. Large neural simulations on large parallel computers. Invited paper at Joint meeting of 5th international conference on bioelectromagnetism and 5th international symposium on noninvasive functional source imaging within the human brain and heart (BEM\&NFSI); May; Minneapolis (MN).

Izhikevich EM. 2005. Unpublished. http://vesicle.nsi.edu/users/izhikevich/interest,

Kepner J. 2004. MatlabMPI. J. Parallel Distrib. Comput. 64, 8 (Aug. 2004), 997-1005. 
Koch H, Van Drongelen W, Marcuccilli CJ, Ramirez J.-M. 2003. Are slow oscillations in somatosensory cortex of mice in vitro dependent on the persistent sodium current? Society for NeuroscienceWashington, DC.Society for Neuroscience. Abstract 411.18.

Krimer LS, Goldman-Rakic PS. 2001. Prefrontal microcircuits: membrane properties and excitatory input of local, medium and wide arbor interneurons. J Neurosci 21:3788-3796.

Leigh J, De Schutter DE, Lee M, Bhalla US, Bower JM, DeFanti TA. 1993. Realistic modeling of brain structures with remote interaction between simulations of an inferior olivary neuron and a cerebellar Purkinge cell. In: Proceedings of the SCS simulations multiconference; March; Arlington (VA).

Mathworks.2005. http://www.mathworks.com/.

Migliore M, Cannia C, Lytton WW, Markram H, Hines ML, Parallel network simulations with NEURON. J Comput Neurosci May 26.

Mohr B, Brown D, Malony A. 1994. TAU: a portable parallel program analysis environment for pC++. In: Proceedings of CONPAR 94 - VAPP VI, University of Linz, Austria, LNCS 854; Sept.; p. $29-40$.

Mountcastle VB. 1997. The columnar organization of the neocortex. Brain 120:701-722.

Nieuwenhuys R. 1994. The neocortex. an overview of its evolutionary development, structural organization and synaptology. Anat Embryol 190:307-337.

Nunez PL. 1981. Electric fields of the brain: the neurophysics of EEG. Oxford: Oxford University Press. 
ParaView. 2005. http://www.paraview.org.

pGENESIS. 2005. http://www.genesis-sim.org.

Pumain R, Menini C, Heinemann U, Louvel J, Silva-Barrat C. 1985. Chemical synaptic transmission is not necessary for epileptic seizures to persist in the baboon Papio papio. Exp Neurol 89:250-258.

Tau.2005. http://www.cs.uoregon.edu/research/tau/home.php.

Tutte, WT. 1963. How to draw a graph. Proceedings London Mathematical Society 13(3):743768.

Van Drongelen W, Hereld M, Lee HC, Papka ME, Stevens RL. 2002. Simulation of neocortical activity. Epilepsia 43, Suppl. 7:149.

Van Drongelen W, Lee HC, Hereld M, Jones D, Cohoon M, Elsen F, Papka ME, Stevens RL. 2004a. Simulation of neocortical epileptiform activity using parallel computing. Neurocomputing 58-60:1203-1209.

Van Drongelen W, Lee HC, Koch H, Elsen F, Carroll MS, Hereld M, Stevens RL. 2004b. Interaction between cellular voltage-sensitive conductance and network parameters in a model of neocortex can generate epileptiform bursting. Invited presentation; IEEE Catalog 04CH37558C: 4003-4005a.

Van Drongelen W, Koch H, Marcuccilli CJ, Viemari J, Tryba AK, Loweth JA, Ramirez J.-M., Elsen F. 2004c. Persistent sodium current plays a critical role in the generation of slow 
oscillations in in vitro neocortex brain slices of mice and humans. 2004 Society for Neuroscience annual meeting.

Van Drongelen W, Lee HC, Koch, Hereld M, H, Elsen F, Chen Z, Stevens RL. 2005a. Emergent epileptiform activity in neural networks with weak excitatory synapses. IEEE Trans. Neur Sys \& Rehab13:236-241.

Zador A, Koch C, Brown TH. 1990. Biophysical model of a Hebbian synapse. Proc Natl Acad Sci U S A. 87:6718-6722. 

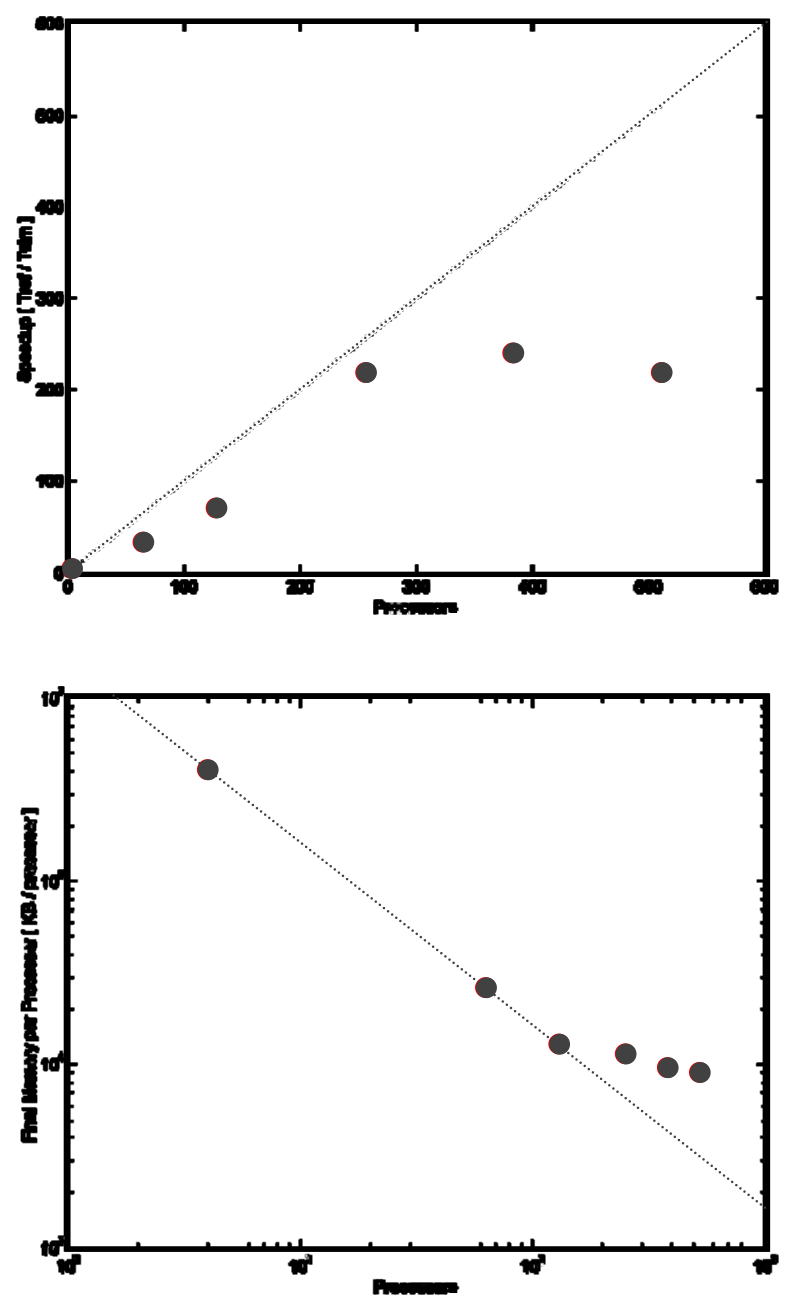

Fig. 1

Scaling study of our development code, pNeo, running on Blue Gene. The size of the simulation is held fixed while the number of processors allocated is changed. The top panel shows speedup as a function of number of processors. Beyond 256 processors the communication time begins to dominate the total execution time, resulting in less than linear speedup. We expect that simulations involving more cells would enjoy linear speedup on larger allocations of processors. The bottom panel shows required memory per processor scales with number of processors. Again we see that for hundreds of processors the problem shows signs of mismatch to the available resources. 

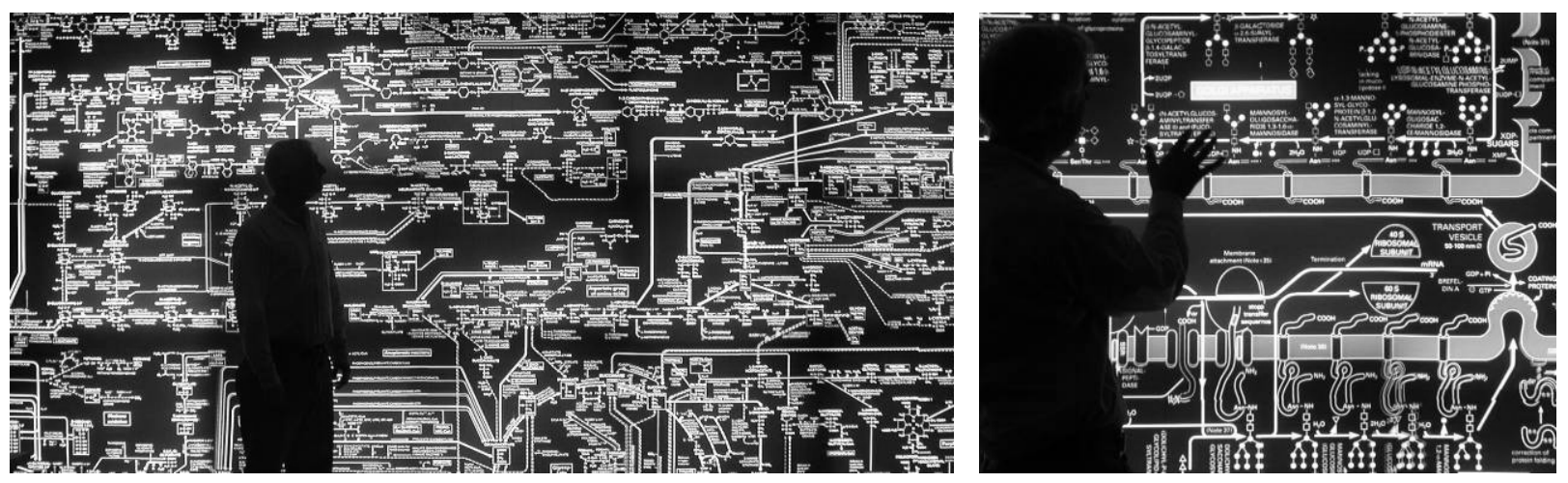

Fig. 2

Initial experiment in large-format high-resolution interactive visualization - in this case a visualization of metabolic pathway diagrams. The diagram at native resolution is larger than the 12 million pixel ActiveMural, physically 16 feet wide by 8 feet tall. 


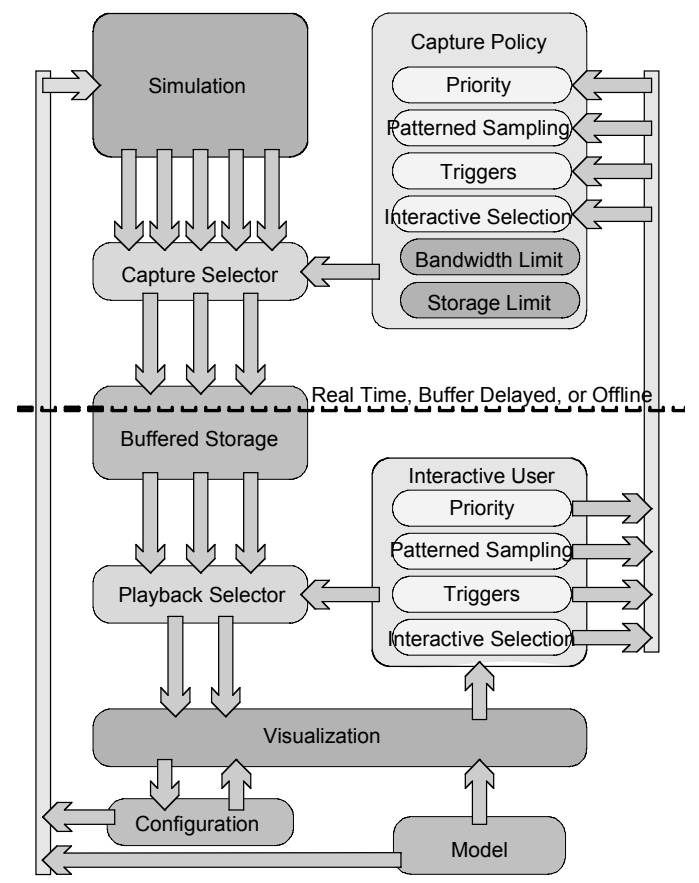

Fig. 3

Architectural diagram of the proposed interactive neural simulation environment. 


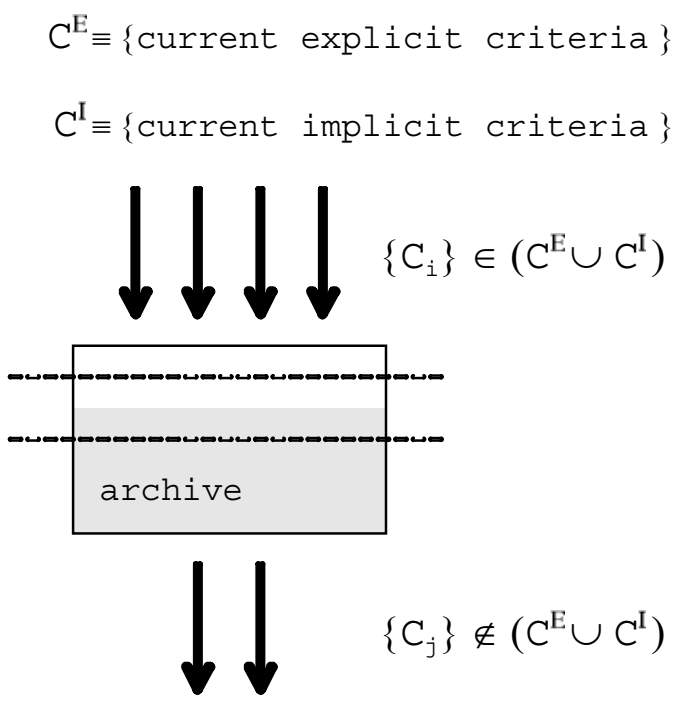

Fig. 4

Illustration of leaky bucket streaming data storage model. The box represents total available disk space. High and low water marks (indicated as dashed lines) determine behavior of the fill and empty processes that regulate data archiving. 


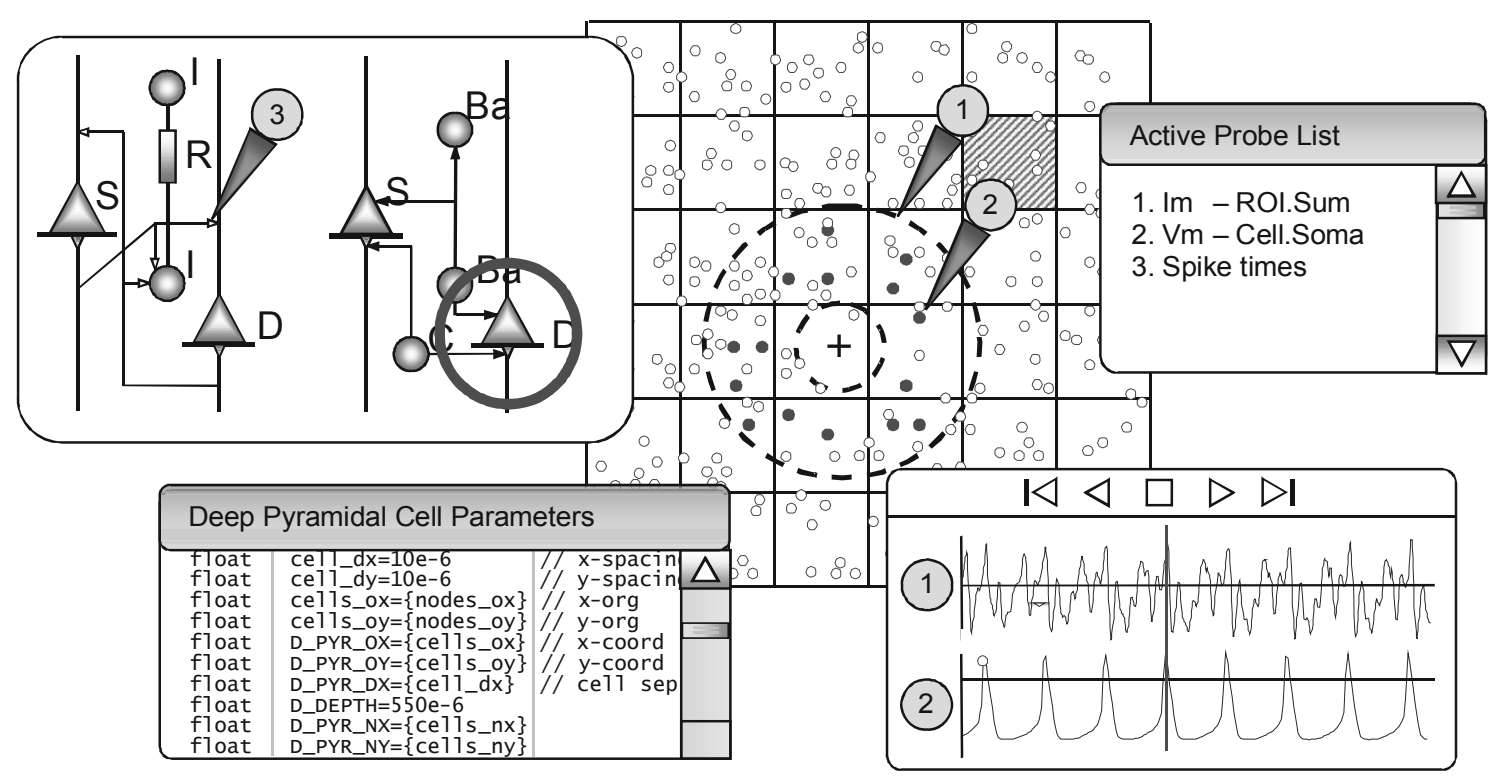

Fig. 5

Mockup of possible visual depiction of the neocortex model employed as markup space for simulation configuration, active probe deployment, and signal monitoring. The schematic on the left show the wiring diagram for excitatory and inhibitory connections. The middle panel indicates a 2D layout of cells in a patch of the model with an annular region of interest, including rectangular a grid denoting which processor in the cluster computes which portion of the patch. Recorded or real-time traces of probed quantities are at the bottom right. 
Table 1. Simple MACRO Instrumentation Example

\begin{tabular}{|c|c|}
\hline The macro & PROBE_2D PROBENAME ARRAYNAME I J \\
\hline $\begin{array}{l}\text { Would be inserted into } \\
\text { the code to expose } \\
\text { intermediate results: }\end{array}$ & $\begin{array}{l}\mathrm{v}(i, j)=\text { someThing; } \\
\text { PROBE_2D ActionPotential } \mathrm{v} i j \\
\mathrm{v}(i, j)=\text { someThingElse; }\end{array}$ \\
\hline And would expand to: & $\begin{array}{l}\mathrm{v}(i, j)=\text { someThing; } \\
\text { if }\left(\mathrm{AP}_{\mathrm{A}} \text { FLAGS }\left[\_\mathrm{AP} \_ \text {ActionPotential }\right]\right)\{ \\
\quad\} \\
\mathrm{v}(i, j)=\text { someThingElse; }\end{array}$ \\
\hline $\begin{array}{l}\text { It would ensure creation } \\
\text { of the flag array and add } \\
\text { symbols }\end{array}$ & $\begin{array}{l}\text { boolean _ }{ }^{\mathrm{AP}} \_ \text {FLAGS }[] \\
\text { \#define _ } \mathrm{AP} \_ \text {ActionPotential }={ }^{\mathrm{APX}} \text { _INDEX } \\
\text { \#define__APX_INDEX }+=1\end{array}$ \\
\hline
\end{tabular}


Hereld et al.

Interactive Million-Neuron Simulation

The submitted manuscript has been created by the University of Chicago as Operator of Argonne National Laboratory ("Argonne") under Contract No. W-31-109-ENG-38 with the U.S. Department of Energy. The U.S. Government retains for itself, and others acting on its behalf, a paid-up, nonexclusive, irrevocable worldwide license in said article to reproduce, prepare derivative works, distribute copies to the public, and perform publicly and display publicly, by or on behalf of the Government. 